\title{
Agent-based hantavirus transmission model incorporating host behaviour and viral shedding heterogeneities derived from field transmission experiments
}

\author{
Molly Kaplan ${ }^{a}$, Carrie A. Manore ${ }^{b}$ and Karoun H. Bagamianc \\ ${ }^{a}$ School of Public Health and Tropical Medicine, Tulane University, New Orleans, LA, USA; ${ }^{b}$ Department of \\ Mathematics, Center for Computational Science, Tulane University, New Orleans, LA, USA; ${ }^{c}$ Department of \\ Environmental and Global Health, Emerging Pathogens Institute, University of Florida, Gainseville, FL, USA
}

\begin{abstract}
Behavioural and environmental heterogeneities among host populations can play an important role in hantavirus transmission. We designed an agent-based model to determine the relative role of direct and indirect transmission on Sin Nombre hantavirus (SNV) dynamics in deer mice, incorporating host heterogeneities. We parameterized the model to reproduce aggressive encounters, movement and excretions from field-based studies and lab experiments. Our model captured known properties of SNV spread and matched the outcomes of transmission experiments. Although the model was not fit to $R_{0}$ values, the $R_{0}$ distribution from our simulations was similar to values from other hantavirus models. We also found that a small per cent of mice were responsible for a high per cent of direct transmission. Our model indicated that mouse heterogeneity and environmental contamination are both important. Model extensions can explore larger ecosystem dynamics by incorporating temporal heterogeneity, to understand how changes in host characteristics and environment influence SNV transmission.
\end{abstract}

\section{ARTICLE HISTORY}

Received 16 May 2016

Accepted 26 September

2016

\section{KEYWORDS}

Individual-based model; Sin Nombre hantavirus; deer mouse; viral shedding; direct transmission; indirect transmission

\section{Introduction}

Hantaviruses are negative strand RNA viruses that are endemic in rodent populations worldwide (Jonsson, Figueiredo, \& Vapalahti, 2010); possible hosts also include bats, shrews and moles (Yanagihara, Gu, Arai, Kang, \& Song, 2014). Some hantaviruses are pathogenic for humans and are usually transmitted to humans by inhalation of aerosolized virus from rodent saliva and excreta. One such example is Sin Nombre virus (SNV), which is responsible for the majority of hantavirus pulmonary syndrome in humans across North America (Mills, Amman, \& Glass, 2010). Hantavirus pulmonary syndrome has a 37\% fatality rate and no vaccine or cure (CDC Hantavirus website, 2016). The deer mouse Peromyscus maniculatus, a primary host of SNV, is a ubiquitous rodent often found near human dwellings in rural areas (Mills \& Childs, 1998; Mills et al., 2010). Since human cases

CONTACT Carrie A. Manore cmanore@tulane.edu

(c) 2016 The Author(s). Published by Informa UK Limited, trading as Taylor \& Francis Group.

This is an Open Access article distributed under the terms of the Creative Commons Attribution License (http://creativecommons.org/ licenses/by/4.0/), which permits unrestricted use, distribution, and reproduction in any medium, provided the original work is properly cited. 
of hantavirus pulmonary syndrome are most often caused by contact with primary animal hosts or their excreta, understanding transmission and risk patterns in primary hosts can serve to help reduce risk of human disease.

Early models of transmission dynamics predicted that population density was the driving force of directly transmitted pathogens (see e.g. McCallum, Barlow, \& Hone, 2001). However, this relationship is complex, and no direct association between host population density and infection prevalence has been determined for hantavirus (Bagamian et al., 2012a), although mathematical and statistical models have shown a delayed density relationship between population densities and antibody prevalence in some deer mouse populations (Luis, Douglass, Mills, \& Bjørnstad, 2014; Madhav, Wagoner, Douglass, \& Mills, 2007; Mills, Ksiazek, Peters, \& Childs, 1999), and this relationship may be more apparent at regional scales than local scales (Carver, Trueax, Douglass, \& Kuenzi, 2011). The current view that SNV transmission occurs primarily through direct contact is evidenced by the strong correlation between being SNV antibody positive and the presence of wounds (Clay, Lehmer, Previtali, and Jeor, 2009; Douglass et al., 2001). Studies have found peak incidences of both transmission events (TE) and number of new wounds to coincide with the beginning of the breeding season (Bagamian et al., 2012a), which is a time when wild mice display the most aggressive behaviors (Millar, 1989). For SNV to be transmitted through aggressive encounters between mice, a mouse that is uninfected must come into contact and then engage aggressively with an infected mouse. Therefore, deer mice that exhibit more aggressive behaviors have a higher probability of being infected with SNV (Dearing, Clay, Lehmer, and Dizney, 2015; Dizney \& Denise Dearing, 2013).

One study done in an outdoor enclosure found that $40 \%$ of deer mouse interactions were aggressive, including fighting and chasing, and, at the end of their stay in captivity, mice deemed 'bold' were more likely to be SNV positive (55\%) than mice deemed 'shy' (18.6\%) (Dizney \& Denise Dearing, 2013). This heterogeneity among hosts indicates that not all individuals in a population have the same chance of becoming infected by or transmitting the pathogen, due to variations in their susceptibility and their exposure, as well as their innate characteristics (Clay et al., 2009). Woolhouse et al. (1997) proposed that transmission patterns of many vector-borne parasites follow the $80 / 20$ rule, where $20 \%$ of the host population contributes at least $80 \%$ of the net transmissions within a system (Woolhouse et al., 1997). This may also be a good rule of thumb for hantavirus transmission in mice due to the prominent role of aggressive encounters in transmission and the characteristics of individual mice that make a certain minority of the population responsible for the majority of the disease spread. A recent study provided support for this rule, in that it demonstrated heterogeneity among deer mice in both frequency and duration of contacts with other mice (Clay et al., 2009). Throughout captivity in foraging arenas, a minority of the mouse population was responsible for the majority of the contacts. Adler, Clay, and Lehmer (2008) identified three forms of heterogeneity in their contact-heterogeneity model and quantified their effect on prevalence of SNV infection and seroconversion: sex, susceptibility and encounters. Their model indicated that the persistence of SNV in deer mouse populations with a low prevalence results from a combination of heterogeneity in encounters, susceptibility, and mortality that favours the survival of the virus. The model indicates that there are higher prevalence and seroconversion rates in males, who are more likely to be aggressive due to breeding condition. Other markrecapture studies show this male bias in seroconversion (Douglass, Calisher, Wagoner, \& 
Mills, 2007), antibody prevalence (Douglass et al., 2001; Kuenzi, Douglass, Bond, Calisher, \& Mills, 2005) and viral RNA detected in blood samples (Bagamian, Towner, Mills, and Kuenzi, 2013), and this is often linked to wounding (a measure of aggression) (Bagamian et al., 2012b; Douglass et al., 2001; Kuenzi et al., 2005).

In addition to direct transmission, it may be that deer mice can become infected by SNV through exposure to infectious pathogens in their environment. Ecological studies from another natural hantavirus-host system (Puumala virus (PUUV)-bank vole) have linked PUUV prevalence in bank voles to variables associated with facilitating indirect transmission (Linard, Tersago, Leirs, \& Lambin, 2007). Also, laboratory studies of other hantaviruses have shown that rodent hosts are able to become infected indirectly (Gavrilovskaya et al., 1991; Kallio et al., 2006) or through an aerosol route (Yun \& Others, 1999). Understanding the role indirect transmission and virus shedding into the environment is also important in the context of human exposure to SNV (Cline, Carver, \& Douglass, 2010; Richardson, Kuenzi, Douglass, Hart, \& Carver, 2013), which is almost always indirect and hints at possible indirect transmission among mice as well. Once a host is infected, it will remain infected and capable of shedding virus into its environment, often through urination, defecation, and shedding of saliva (Kuenzi et al., 2005; Voutilainen et al., 2015). Virus can survive for a couple of days outside its host, and excreta scent marks are attractive to territorial mammals such as deer mice. This increases the probability that a foraging rodent will explore these areas. Urine secretions can mix with soil, water or other surfaces and the contaminated surfaces become areas where new infections can take place without the presence of the infectious host (Sauvage, Langlais, Yoccoz, \& Pontier, 2003).

Due, in part, to the difficulty in measuring contacts between rodent hosts, as well as the difficulty of simulating and observing wild-like conditions, little is known about the patterns of heterogeneity in directly transmitted zoonotic infections such as SNV. Using data and simulating conditions from the four enclosure experiments done by Bagamian et al. (2012a), and defining parameters based on other studies on SNV in deer mice, we created an agent-based (or individual-based) model to qualitatively mimic transmission in an enclosure. We first designed a simple random walk mouse behavior model that would reproduce known characteristics relevant to transmission, namely, relative day/night movement rates and urination/defecation rates. We then incorporated aggressive encounters and indirect transmission from contaminated surfaces into the model along with variation in these transmission modes corresponding to heterogeneity in viral shedding and aggression in individual mice. The primary goal of this model is to qualitatively assess the following questions: (1) what are the relative contributions of direct and indirect transmission of hantavirus transmission in deer mice? and, (2) what is the role of heterogeneity among hosts in SNV transmission? Our model is designed to ultimately take observed results in the field and lab, and apply them on larger spatial and temporal scales with multiple model trials in order to understand both stochasticity and consistent underlying patterns in SNV spread in the wild (Carver et al., 2015).

\section{Description of the model}

We designed an agent-based model (ABM) from a bottom-up perspective, first fixing simple mouse behavior properties (day and night movement rates and urination/ defecation rates) based on the literature. Our goal was to build the simplest possible ABM 
Table 1. Free parameters and their chosen values. The values were chosen based on a sensitivity analysis as described in Section 3.1.

\begin{tabular}{lc}
\hline Model parameter & Assigned probability \\
\hline Bite probability for aggression level 0 & .03 \\
Bite probability for aggression level 1 & .06 \\
Bite probability for aggression level 2 & .09 \\
Direct transmission probability for infectiousness level 0 & .3 \\
Direct transmission probability for infectiousness level 1 & .6 \\
Direct transmission probability for infectiousness level 2 & .9 \\
Indirect transmission probability for infectiousness level 0 & .003 \\
Indirect transmission probability for infectiousness level 1 & .006 \\
Indirect transmission probability for infectiousness level 2 & .009 \\
\hline
\end{tabular}

that naturally reproduced transmission properties of a small-scale wild system (Bagamian et al., 2012a) while fixing as many parameters and behaviors as possible apriori. The reason for this approach is that if the bottom-up model reproduces enclosure-level transmission dynamics without overfitting, it is likely that the model is capturing the necessary dynamics to move forward at a larger scale. Therefore, the only criteria we used to determine the free parameter values (transmission parameters) were matching the average number of TE per enclosure and the average number of bites per mouse in an enclosure (Bagamian et al., 2012a).

The model was coded using the NetLogo Wilensky (1999) programming language and depicts a system formed by a specified number of agents (mice) representing deer mice trapped in an outdoor enclosure, analogous to those of Bagamian et al. (2012a). The model assumes that all mice operate in a similar way in terms of their daytime vs. nighttime movement patterns, as well as their urination and defecation habits (Table 1). In the model, mice move continuously during the night by the process of choosing a random discrete value from 0 to 359 and turning right this number of degrees, then moving forward one space in that direction. During the day, they follow the same pattern of motion, but at a 90\% slower rate. This reflects the behaviors of North American deer mice, which are active during the night and do most of their resting during the day (Baumgardner, Ward, \& Dewsbury, 1980; Eisenberg, 1962).

Each mouse is randomly assigned a level of aggression as well as a level of infectiousness on a scale of discrete values between 0 and 2, with a uniform distribution. An associated probability of transmission was postulated for and assigned to each level of aggression and infectiousness (Table 2). Levels of aggression and infectiousness are both measures of heterogeneity among individual mice, and are characteristics unique to mice based on various factors such as genetics, stress levels, sex, breeding condition, health, and more. High, medium and low levels of aggression and infectiousness serve as proxies for the wide scale of unique characteristics that would lead to probability of biting as well as virus levels present in the blood and associated shedding rates.

In their review of current literature, Douglass et al. (2007) presented the following: (1) after experimental infection, viral levels in mouse saliva are not always uniform, (2) in regards to tissues involved and degree of replication, persistently infected mice have a large degree of variation and (3) SNV RNA is amplified from tissues and excreta of some, but not all, naturally infected deer mice. The implication of these observations is that there is a degree of variation of viral shedding due to individual differences in mice 


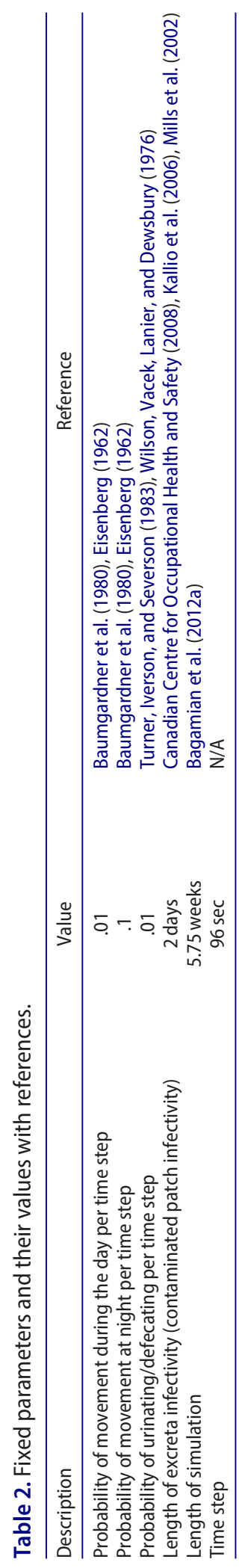


(Douglass et al., 2007). This is incorporated in the infectiousness term in the model and assumes that viral load is directly related to viral shedding and infectiousness.

We followed the ABM description ODD protocol outlined in Grimm et al. (2010).

\subsection{Purpose}

The purpose of this $\mathrm{ABM}$ is to simulate hantavirus transmission in wild deer mice in an enclosure in order to understand the relative contributions of direct and indirect transmission as well as the role that individual heterogeneity plays in transmission. The model reproduces several observed features of mice in captivity such as urination/defecation and relative day and night activity rates. Transmission rates depend on the level of aggression and the level of infectiousness of each individual mouse. The model will provide insight into the interaction of individual mouse behavior (aggression, movement, urination/defecation) and infection characteristics (viral load or infectiousness) with hantavirus disease dynamics, particularly in the context of the relative roles of indirect and direct transmission. The model will be extended to expand from small, enclosed populations to real-world, large-scale scenarios.

\subsection{Entities, state variables, and scales}

The model entities are mice, patches (or cells) and global variables. Time is measured in seconds, and one time-step is $96 \mathrm{sec}$; one day is 900 time-steps. Each simulated enclosure has 1089 patches which, if compared to the .1 ha enclosures in Bagamian et al. (2012a), corresponds to 9.88 square feet per patch (each patch is about $3 \times 3 \mathrm{ft}$ ).

The state variables associated with mice are infected ('sick', true or false), aggression level $(0,1$, or 2$)$, infectiousness level $(0,1$, or 2$)$, a unique identifier for each mouse $(0, \ldots, n-1$ for $n$ total mice), aggression probability (assigned by user for each aggression level), infectious probability (assigned by user for each infectiousness level), time infected (in time-steps), direct transmission (true or false), infected by whom (identifier of transmitting mouse), number of contacts with other mice (occupying the same patch), number of bites from infected mice ('newbites', non-negative integer), colour (red for infected and grey for susceptible) and location (patch identifier).

The state variables associated with patches are colour (black for night, brown for day, yellow if infected), infected ('urinated on', true or false), and time since infected ( 0,1 , or 2 days).

The global variables are day (true for day or false for night), time colour (assigns colours based on day/night cycle), a aggression/bite probability for the currently considered infected mouse, $b$ aggression/bite probability for currently considered susceptible mouse, $c$ transmission probability for infected mouse being considered, 'infector' (mouse responsible for direct transmission), and 'my-slider'.

\subsection{Process overview and scheduling}

We programmed our model in the NetLogo Wilensky (1999) agent-based modelling language (the code and model are original). To summarize, mice in the model move randomly at different rates depending on the time of day and have aggressive encounters 
with each other depending on the aggression parameters of mice occupying the same patch. If one of the mice is infected and an aggressive encounter occurs, then the uninfected mouse has a probability of becoming infected based on the infectiousness level of the infected mouse. Finally, infected mice can contaminate a patch for two days via urine or feces, and uninfected mice can be infected by contact with a contaminated patch.

Each run is 36,226 time-steps and represents 5.75 weeks of capture. One day, therefore, is 900 time-steps (1.6 min per time step), and the environment changes from day to night, every 450 time-steps. Upon set-up of each simulation, $n$ agents (mice) are introduced and their state variables are defined. One of the mice, selected at random, is then infected and its colour changed from grey to red. The simulation starts at night and there are no initially contaminated patches. The boundary conditions are reflective to create an enclosure environment.

We note that NetLogo refers to agents as 'turtles'. Once the 'go' button is clicked, the observer commands the following subprogrammes in order: move agents ('move-turtles'), 'update-display', patch contamination by urination/defecation ('urinate-turtles'), 'directinfect', indirect infect ('patch-infect'), clear patch ('dry-patch'), and, if the number of time-steps are greater than 36226 (5.75 weeks), stop the simulation. These commands are followed at every time step.

Agents decide if to move at every time-step based on whether it is day or night and then choose a random direction in which to move. Once the mice move, the display is updated to reflect whether it is day or night. Mice urinate/defecate (and shed virus if infected) one out of 100 time-steps, corresponding to once every $2.5 \mathrm{~h}$. The urination/defecation probability was estimated based on lab studies and assumptions about excreta volume and conditions needed to successfully contaminate a patch with viable virus. Contamination with infected excreta causes the patch to become 'contaminated' (turn yellow), and each contaminated patch remains infectious for two days. The programme then asks all infected mice if they are sharing a patch with an uninfected mouse. If so, it is decided if a bite will occur based on the aggression level of the infected mouse and the aggression level of the susceptible mouse. If a bite occurs, then transmission occurs (or not) based on the infectiousness level of the infected mouse. The infector and time of infection is noted as well as mode of transmission (direct). If any susceptible mice occupy a contaminated patch, the mouse has a probability of becoming infected based on the infectiousness level of the mouse who contaminated the patch. Time of infection, infector, and mode of infection (indirect) is noted if transmission occurs. Finally, any patches that have been infected for two days are set back to uninfected.

\subsection{Design concepts}

\subsubsection{Basic principles}

Our general modelling approach was to balance simplicity with heterogeneity of individuals. Therefore, we used a simple random walk for mouse movement with movement rates dependent on time of day and reflective of observed movement rates of mice in captivity and the wild. Similarly, we assumed that urination/defecation occurs randomly every $2.5 \mathrm{~h}$ or so, also reflecting observations in the field and captivity.

We allowed for more complexity in mouse aggression, viral load and indirect/direct transmission probabilities. Rather than have mice sense each other while moving and 
complicating the movement procedure, we fixed aggression levels for mice occupying the same cell such that a reasonable range of bites occur based on the field study in Bagamian et al. (2012a). We were also concerned about overfitting since there were not enough data to properly constrain the nine free parameters (three aggression levels, three direct infectiousness levels, and three indirect infectiousness levels). So, we somewhat arbitrarily decided that each aggression and infectiousness level would be a factor of 3 (low), 6 (medium) and 9 (high).

\subsubsection{Emergence}

We wanted to observe transmission dynamics as they would emerge from these simple behaviors of moving, urinating/defecating, and biting, so we fixed all parameters except aggression and infectiousness apriori based on the literature. We then fit the free parameters mentioned above by exploring the parameter space and choosing the set of parameter values that reasonably reproduced (a) the average number of bites per mouse and (b) the average number of TE per enclosure as observed in Bagamian et al. (2012a). As such, other outputs of the simulations such as relative contributions of direct transmission vs. indirect transmission, the distribution of the number of direct TE per any given infected mouse, and the reproduction number distribution were considered to be emergent properties of the model. These outputs did not in any way determine parameter values chosen but were instead observed and compared to the literature post-simulation.

\subsubsection{Adaptation}

The model entities currently have no adaptive traits.

\subsubsection{Objectives}

Since there are no adaptive traits, there are no objectives governing these traits.

\subsubsection{Learning}

There is no learning in this model.

\subsubsection{Prediction}

Agents do not predict any future conditions or consequences of actions in this model.

\subsubsection{Sensing}

Mice sense the patch they are occupying and whether or not another mouse is in it and the time of day, which determines movement rates.

\subsubsection{Interaction}

Mice can interact with each other via aggressive biting. They interact with the environment by urinating/defecating on a patch and potentially contaminating that patch with infectious material. Susceptible mice may become infected by occupying a contaminated patch.

\subsubsection{Stochasticity}

Mice are assigned characteristics to do with aggression and viral load randomly (uniform distribution) and independently. Mouse movement and urination/defecation are random. Similarly, whether bites occur or transmission occurs are random events based on the characteristics of the infectious and susceptible mice being considered. We use stochasticity to capture the wide range of occurences observed in the enclosure experiments we considered and observed in the wild. 


\subsubsection{Collectives}

There are no collectives in the model.

\subsubsection{Observation}

We collected data on all TE including associated time of infection, whether or not transmission was direct or indirect, and which agent was responsible for transmission. We also kept track of the number of bites from infected mice that each mouse got during the simulation and the total number of pairwise contacts (when mouse is in the same patch as another mouse). Additionally, each mouse's aggression and infectiousness level were included in the final output.

\subsection{Initialization}

Upon set-up of each simulation, $n$ agents (mice) were introduced to random locations in the enclosure, and their characteristics are defined. We set $n=20$ for every simulation presented here to replicate a small enclosure. Each mouse was assigned an aggression level and a level of infectiousness at random (uniform distribution), from discrete numbers 0-2 representing low, medium, and high infectiousness or aggression. All mice were initially uninfected, setting their infection status to 'false'. One of the $n$ mice, selected at random, was infected, setting its infection status to 'true', changing its colour from grey to red, and setting its infected by whom variable to 100 so that we can tell it was a donor mouse. The day variable was initially set to 'false', so the simulation starts at night and the patches start out black, and infected (or urine present) was set to 'false' for all patches so there were no initially contaminated patches.

\subsection{Input data}

The model does not currently input from external sources.

\subsection{Submodels}

Model flow and use of submodels can be found in Figure 1. The 'move agents' procedure controls mouse movement. If it is night, then a floating number less than 100 is randomly generated, and if it is less than 10, the mouse moves right at a randomly chosen angle and forward 1 cell (so mice move about $10 \%$ of the time). If it is day, the mice are moved in the same way, but only if a floating number out of 100 is less than 1 (moving $1 \%$ of the time). The 'update-display' command controls the colour of the patches, based on a loop module that cycles 900 time-steps, which is equivalent to a 24 -h day. If the time-steps in the loop is greater than 450 , it is daytime, and the patches that are not 'urinated-on' are set to brown; otherwise patches that are not 'urinated-on' are set to black. 'Count-contacts' keeps a running tab for each mouse of every time they occupy the same patch as another mouse, by asking each mouse on every time-step if there are any other agents in the cell, and if so, adding +1 to their contact count.

The 'direct-infect' command loops over all infected mice and assigns to parameter $a$ a bite probability for each infected mouse at the current time, which corresponds to their aggression levels (see Table 1). The variable 'infector' is set to the infected mouse's unique 


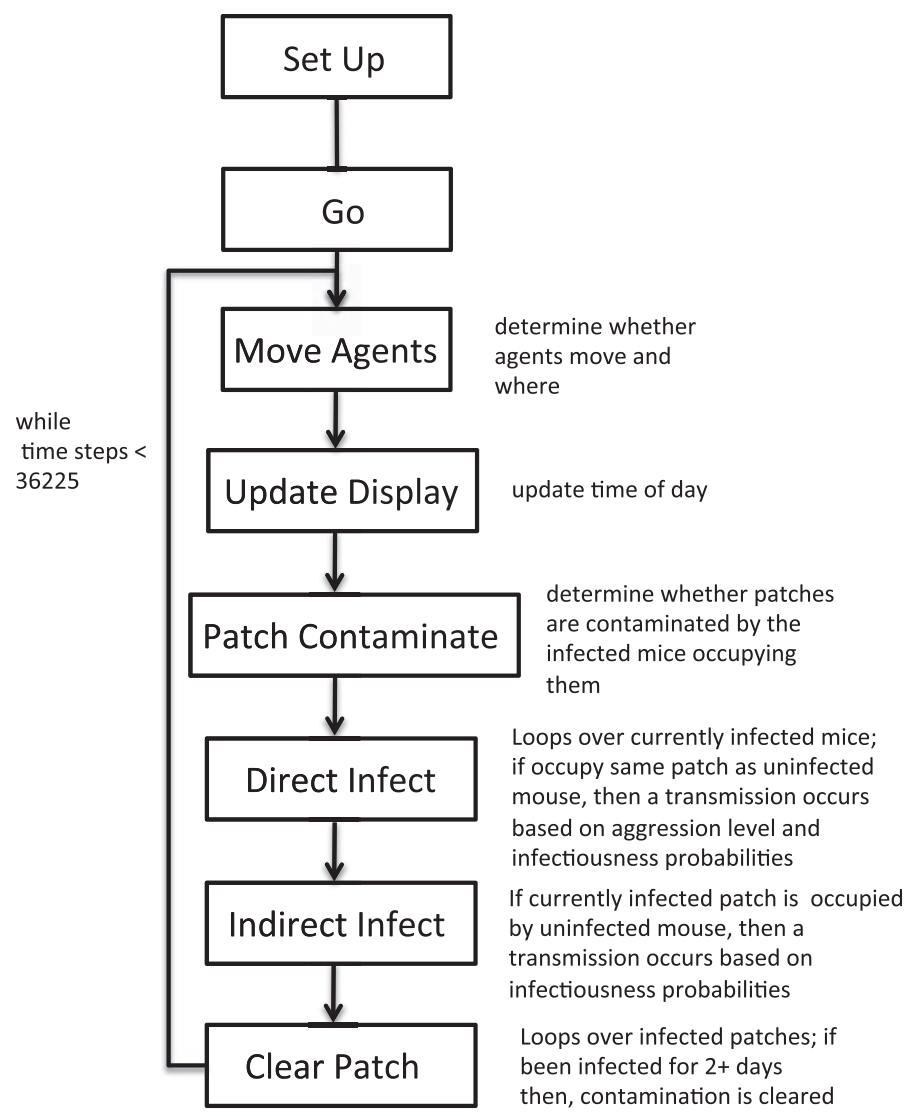

Figure 1. Flow chart of model simulation procedures with submodels listed.

identifier. This allows the model to track who is potentially infecting whom. Then, if another mouse that is uninfected is occupying the same patch, a second parameter $b$ is set equal to the uninfected mouse's bite probability based on their aggression level. If a random floating-point number between 0 and 100 is less than $a \times b$, an aggressive encounter between the infected and uninfected mouse has occurred (i.e. a bite), and the uninfected mouse's variable number of bites by infected mice ('newbites') increases by 1 . This tracks the number of times a susceptible mouse has an aggressive encounter with an infected mouse. Finally, a third value $c$ is defined as the transmission probability corresponding to the infectiousness factor of the infected mouse (see Table 1). If the uninfected mouse has an aggressive encounter with the infected mouse and a random floating point number in the interval $[0,100]$ is less than $c$, the 'get-infected' procedure takes place for the uninfected mouse. In this case, the newly infected mouse's infected by variable 'infectby' is set to 'infector', 'direct' is set to true (meaning a direct transmission occurred), and the time-step at which the event occurred is noted (time infected).

The patch contamination by 'urinate-turtles' procedure sets a patch to 'contaminated' if it is occupied by an infected mouse and a randomly chosen floating number less than of 100 is less than 1 (i.e. urination/defection occurs every $2.5 \mathrm{~h}$ on average); the patch colour is 
changed to a shade of yellow when contaminated. The shade is based on the infectiousness of the mouse. 'Infector' is set to the identifier of the mouse, and 'urinated-on' is set to 'true' for the patch, a countdown is set to 1800 time-steps (2 days), and a new variable 'pinfector' is set to the value in 'infector'. These contaminated patches dry when the 1800-time-step countdown expires using the clear patch procedure that asks the patches upon every timestep if the countdown is zero, and if so, changes the colour back to normal and the patch to uncontaminated.

The indirect infect ('patch-infect') command asks mice who are uninfected if they are occupying a patch that has been contaminated. If so, transmission occurs at different probabilities depending on the infectiousness of the mouse that contaminated the patch ('pinfector'). Transmission takes place if a random floating number between 0 and 100 is less than the associated probabilities for infectiousness levels of 0,1 and 2, respectively, of the infector. If transmission occurs, 'direct' is set to false, 'infectby' is set to 'pinfector' and the time-step at which the event occurred is noted.

\section{Model analysis and simulations}

Parameters for this $\mathrm{ABM}$ were derived in part from comparing simulation output with data from the enclosure experiments by Bagamian et al. (2012a) that focused on studying population density and seasonality as they relate to SNV transmission. These experiments took place in the summer and fall of 2007 and 2008 in shrub-steppe grassland near Butte, Montana, USA. Four experiments were conducted in six .1-ha enclosures constructed of sheet metal, each of which contained four underground nest burrows that provided safe, permanent cover for the mice. Predetermined numbers of infected and uninfected adult wild deer mice were released into enclosures and trapped weekly or bi-weekly. Upon recapture, blood samples were collected to test for SNV RNA and antibody, and body weight, breeding condition (scrotal or abdominal testes), trap location, and number of wounds on the ears and tail (as an indicator of aggressive encounters) were recorded. The study found that there was no significant dependence of transmission frequency on population density. SNV transmission was, however, significantly dependent on seasonality, which may be related to more aggressive encounters during breeding season. Taken together, these data imply that levels of aggression and infectiousness may have an important role in transmission. As a result, we used the experimental outcomes, such as TE and number of new wounds to inform our exploration of how varying aggression and unique mice characteristics affect SNV transmission dynamics. Calculated values from the study that we used to inform the model were average per cent of the susceptible mice infected by the end of each trial (25.4\%) and the average number of wounds per mouse, including the donor mouse, (8.97 per mouse) at the end of each trial.

\subsection{Sensitivity analysis and parameterization}

In order to decide on the final parameters, a sensitivity analysis was conducted in which a variety of different parameters for bite probability, direct transmission probability and indirect transmission probability were tested. In the interest of simplicity and to minimize overfitting, the numbers 3, 6 and 9 were used for low, medium and high levels of aggression and infectiousness, respectively, and were varied by orders of magnitude to explore the 
Table 3. Sensitivity analysis and parameter exploration for the free parameter choices.

\begin{tabular}{lccc}
\hline Bite probability & Direct transmission probability & Indirect transmission probability & Avg \# transmissions per run \\
\hline $.003, .006, .009$ & $.03, .06, .09$ & $.003, .006, .009$ & .38 \\
$.003, .006, .009$ & $.3, .6, .9$ & $.03, .06, .09$ & 10 \\
$.03, .06, .09$ & $.03, .06, .09$ & $.003, .006, .009$ & .62 \\
$.03, .06, .09$ & $.03, .06, .09$ & $.03, .06, .09$ & 9.16 \\
$.03, .06, .09$ & $.3, .6, .9$ & $.003, .006, .009$ & 2.67 \\
$.3, .6, .9$ & $.003, .006, .009$ & $.003, .006, .009$ & 2.94 \\
$.3, .6, .9$ & $.03, .06, .09$ & $.003, .006, .009$ & 16.1 \\
$.03, .06, .09$ & $.003, .006, .009$ & $.003, .006, .009$ & 8.55 \\
$.03, .06, .09$ & $.003, .006, .009$ & $.03, .06, .09$ & 11.12 \\
\hline
\end{tabular}

Notes: We restricted low, medium and high $(0,1$, and 2$)$ levels of aggression and infectiousness in mice to corresponding powers of 10 times 3, 6 and 9. Levels are translated into probabilities, so must be between 0 and 1. The parameter combinations not shown can be inferred to be outside our range by direct comparison with the results here.

parameter space. Multiple combinations of bite probability, direct transmission probability and indirect transmission probability were tested (Table 3). As the experimental enclosure study produced an average transmission per cent of $25.4 \%$ over the course of the experiment, a feasible range was determined to be $10 \%-35 \%$ based on the range of transmission in the study $(2.7 \%$ in fall, $24.2 \%$ late summer, $12.5 \%$ mid-summer, and $35.3 \%$ early summer). Other studies in the field give seroprevalence of $15.8 \%$ (Douglass et al., 2001) and 20.2\% (Abbott, Ksiazek, \& Mills, 1999), which are within our chosen range. In our model simulations, there were 19 susceptible mice in each trial (and one donor mouse) so the feasible range of average number of TE per simulation is $1.9-6.65$. Also, the average number of new scars per mouse per enclosure in the experimental study was 8.97 per mouse, with a range from 0 to 39 and a median value of 4 new scars per mouse.

Two parameter combinations resulted in average numbers of TE that were within this range (2.67 and 2.94, see Table 3). The parameters with an average number of TE of 2.94 produced other results that were not feasible. The average number of infected bites per mouse was too high compared with the wound observations from the experimental study with an average of 20.44 bites from infected mice per mouse per run for those parameters. Summarizing the experimental data indicated that the average total number of new wounds per mouse was 8.97, including bites from both infected and non-infected mice. While our model does not currently count every bite (only infectious bites), it can be inferred that the number of new wounds from infected mice would be much lower than total wounds, making the aforementioned parameters unrealistic. The parameters that were decided upon (corresponding to $2.67 \mathrm{TE}$ on average per run) produced an average number of infected bites per mouse of .165, resulting in 3.3 total infectious bites per run on average. Infectious bites are about $1.8 \%$ of the total bites, so each mouse had, on average, 8.6 bites per run for the chosen parameter values. This is close to the experimental average of 8.97. Details of the sensitivity analysis are provided in Table 3 . With data to better constrain these nine transmission-related parameter values, more technical fitting algorithms could be used in the future to determine the parameters.

\subsection{Simulation results}

For the analysis, we ran the model 100 times, with 20 mice in each run. A total of $267 \mathrm{TE}$ occurred over 100 runs of the model, averaging to 2.67 TE per run with a range from 0 


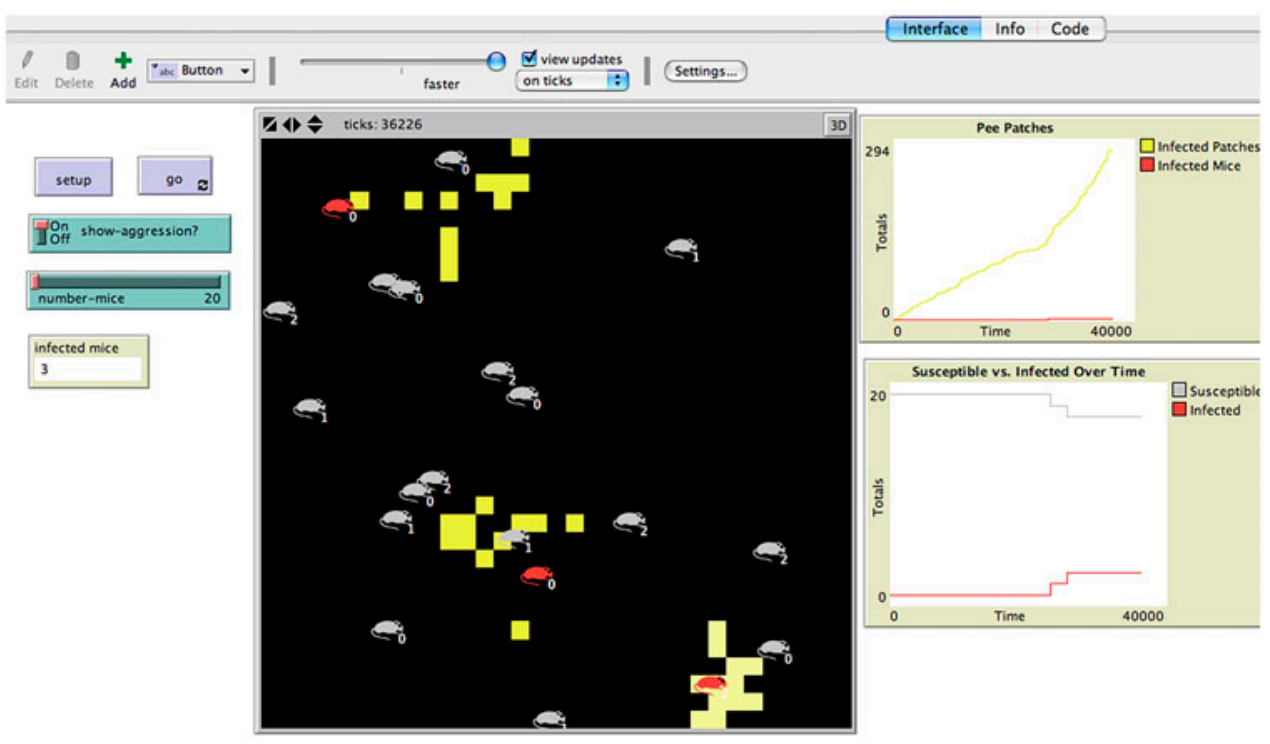

Figure 2. Representative run of the model captured at the end of the run.

Note: Red mice are infected, grey are susceptible, black patches are uninfected, and yellow patches are infected.

to 11 . The number of TE does not include infection of the donor mouse. The number of TE that occurred per week increased as time progressed, with $10 \%$ of TE occurring during week 1 and 24\% of TE occurring during week 5 (week 6 was 3/4 the length of weeks 1-5).

A representative run with model output in NetLogo shows the potential variation in mouse characteristics and transmission (Figure 2). The screen shown is at the end of the model run. For this run, the donor mouse had a high aggression level (2) but low level of viral shedding (0), or infectiousness. The first transmission event was at three weeks and was direct via an aggressive encounter. The newly infected mouse had low aggression level (0) but high viral shedding (2) and the final transmission event at the beginning of week four was indirect via an infected patch. The cumulative number of infected environmental patches (upper right plot) increased more or less linearly with time with the slope increasing after the first transmission event.

The average number of TE that occurred per run increased with both the donor mouse's level of aggression (Figure 3(a)) and infectiousness (Figure 3(b)). Donor mice with higher aggression transmitted more often. Mice with high infectiousness also resulted in more TE. Of the 267 TE that occurred during the 100 trials, 65\% resulted from direct contact through aggressive interactions, and $35 \%$ were through exposure to contaminated infectious patches.

Based on the assumption that increased aggressive nature of rodent hosts increases probability of infection, it was expected that the average aggression level of the uninfected mice would be lower than that of the infected mice. Upon completion, the average aggression level of the uninfected mice was .95 and that of infected mice was 1.21. The average aggression for infected mice does not including the donor mice for each trial, as those are assigned at random and do not relate to aggression level. More aggressive mice 


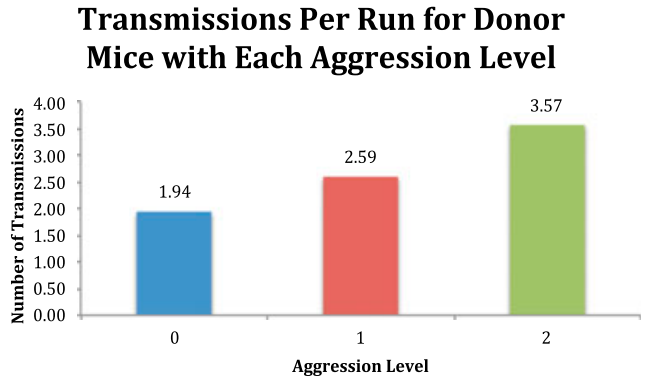

(a) Average number of transmissions per run for donor mouse aggression levels.
Transmissions Per Run for Donor Mice with Each Infectiousness Level

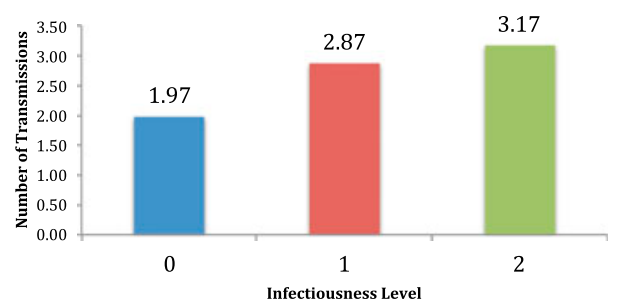

(b) Average number of transmissions per run for donor mouse infectiousness levels.

Figure 3. Transmission events per run for donor mouse aggression and viral load (infectiousness). Notes: For donor mice with aggression level 0 , number of transmissions ranged from 0 to 6 , for aggression level 1 from 0 to 8, and for aggression level 2 from 0 to 11 . For donor mice with infectiousness levels 1 , 2 , and 3 , the number of transmissions ranged from $0-11,0-9$, to $0-11$, respectively.

were more likely to both infect and become infected. This agrees with the result from Bagamian et al. (2012a) that the average number of wounds per mouse for infected mice was 10.4 while the average number of wounds per mouse for uninfected mice was 7.95. Other field studies found that more aggressive mice (and males) are more likely to be SNV positive as well (Abbott et al., 1999; Clay et al., 2009; Douglass et al., 2007).

The 80/20 theory postulates that for some disease systems such as vector-borne diseases, $20 \%$ of individuals are responsible for approximately $80 \%$ of the transmission. Since direct transmission for hantavirus in mice is primarily the result of aggressive contacts, this model produced a similar trend, where a minority of mice was responsible for a majority of the direct TE. Specifically, out of 367 infected mice, including donor mice, only $34.3 \%$ were responsible for at least 1 direct TE. Moreover, 90 mice were responsible for 139 direct TE which is $80 \%$ of the 174 total direct TE. Therefore, roughly $24 \%$ of the mice were responsible for $80 \%$ of direct transmission. Figure 4 shows the frequency distribution of number of direct TE for infected mice. This figure is qualitatively similar to the results of Clay et al. (2009), particularly their Figure 1 showing the frequency distribution of number of contacts per mouse. Recall that we fit parameters to produce the average number of bites per mouse in Bagamian et al. (2012a) only (not the range), so this distribution of direct TE is emergent from the model.

Of the 367 infected mice, including donor mice, and a total of 93 indirect TE, 70 mice were responsible for 1 indirect TE each, 10 were responsible for $2 \mathrm{TE}$, and one mouse was responsible for 3 indirect TE. So, only $22 \%$ of the infected mice were responsible for any indirect transmission. Of the mice that were responsible for an indirect TE, $13.5 \%$ produced $25 \%$ of the indirect TE and $86 \%$ produced $75 \%$ of the indirect TE. Thus, although there is a skew in the number of mice responsible, the $80 / 20$ rule does not appear to hold for indirect transmission. This pattern makes sense because although mice with high infectiousness do shed more virus in a patch, making it more infectious and resulting in a slight skew, contact with contaminated patches by susceptible mice is random. 


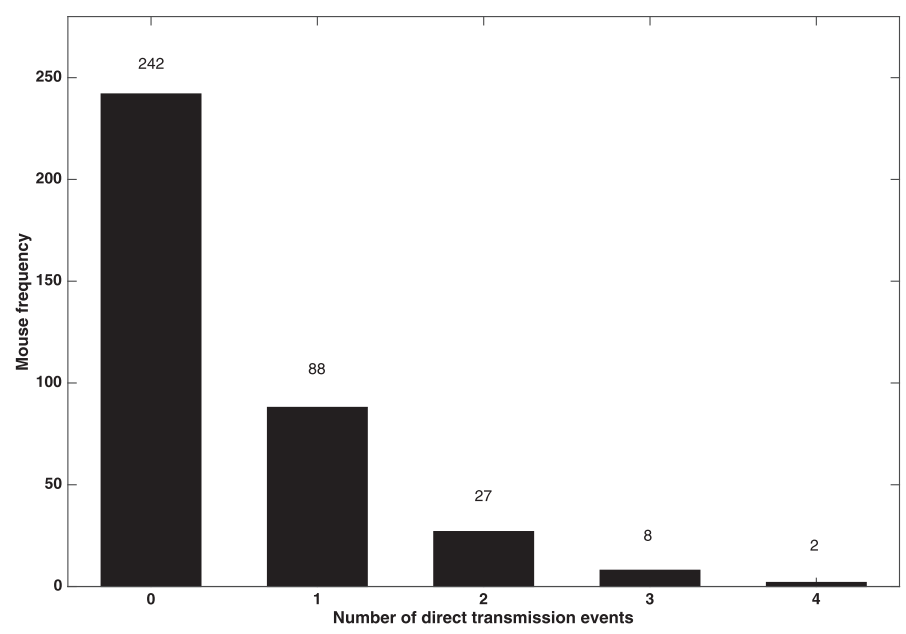

Figure 4. Number of direct transmission events that infected mice were responsible for during the simulation.

\subsubsection{Basic reproduction number}

The basic reproduction number is the average number of new infections from a single infected individual introduced into a fully susceptible population. This value for our model is the average number of mice who become infected as a result of either a direct or indirect $\mathrm{TE}$ from the donor mouse per run and was computed directly from the model output since who infected whom is known for every TE. The basic reproduction number $\left(R_{0}\right)$ calculated from the results of 100 trials was 1.3 , with actual donor mouse reproduction numbers ranging from 0 to 4 . A frequency distribution of the reproduction number is shown in Figure 5. Many donor mice did not transmit at all during the simulation and the majority infected one or two other mice. Rarely, donor mice infected three or four other mice. So, while the basic reproduction number was 1.3 , there was a wide range of possible donor reproduction numbers depending on the donor mouse characteristics and the characteristics of the mice with whom it came into contact. In fact, only about $30 \%$ of the donor reproduction numbers were approximately the same as $R_{0}$ (about 1 ); $45 \%$ were more and $26 \%$ were less.

\section{Discussion and conclusion}

The ABM provides a virtual setting to replicate observed or potential behaviors of a complex system and to test relevant hypotheses with a range of 'what-if' scenarios. By modelling transmission dynamics of hantavirus infection in deer mice and including host heterogeneity, we provide a step towards better understanding these dynamics in the wild and broadening our understanding of rodent-borne zoonotic viruses. Our research contributes to understanding the transmission ecology of SNV by clarifying the relative contributions of direct and indirect transmission of hantavirus and the influence of unique host factors such as viral level and aggression level. Since there are a wide range of behaviors and individual characteristics in wild mice populations, different dynamics than we would expect by only considering mean field often occur. Thus, to understand how 


\section{Frequency of Basic Reproduction Number}

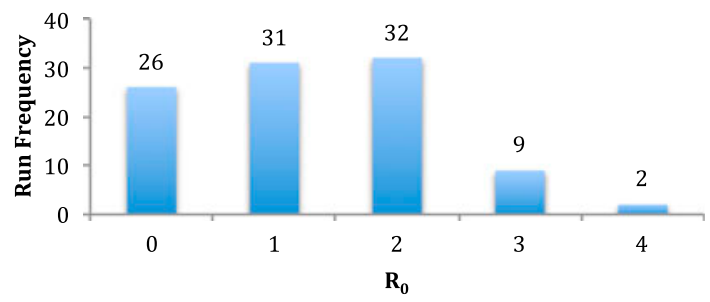

Figure 5. Distribution of the basic reproduction number, $R_{0}$, for the 100 test runs at baseline parameter values.

Note: The basic reproduction number is the number of direct or indirect transmissions for which each donor mouse is responsible.

transmission changes as species distributions and characteristics vary and as environmental conditions vary, we must consider heterogeneities. Knowledge of SNV transmission in its host populations will contribute to development of more accurate models of changing risk to humans and may lead to more effective disease prevention and mitigation at the wildlife-human interface.

To the best of our knowledge, we are the first to develop an ABM to simulate the effect of unique host characteristics on the transmission of hantavirus between rodent hosts. Our research links within-host (viral shedding, aggression) and between-host dynamics. By specifying the behavioral rules of individual mice, and the rules of mice interactions, we can explore the macro-level consequences resulting from these individual-level interactions. Parameter values were chosen based on current literature and by comparing average number of bites and TE to the Bagamian et al. (2012a) field study. An unanticipated property of our simulations was that direct transmission more or less followed thebreak 80-20 rule, so a relatively small per cent of the mice were responsible for a high per cent of direct TE. Indirect transmission was not found to follow this trend, as contact with virus-contaminated land by susceptible mice is random. The $R_{0}$ distribution from our simulations were very close to computed values from other hantavirus mathematical models. This is especially noteworthy as they were not previously chosen metrics, but rather emerged from our simulations based on field transmission experiments.

This model supports current theories that the primary mode of transmission of hantavirus between hosts is direct transmission through aggressive encounters. However, indirect transmission does account for $35 \%$ of TE in our simulation, so it cannot be ignored and is likely a major contributor to viral spillover to humans. Host heterogeneity plays a key role in hantavirus transmission, as the number of TE increased with increased levels of host aggression and infectiousness. The dissimilarity in susceptibility and exposure among hosts lead to a disparity in transmission dynamics and to trends which resemble the 80/20 theory for direct transmission (Figure 4). The role of aggression in transmission suggests that transmission would be significantly reduced if fewer mice were highly aggressive. In fact, recent research shows that complex and diverse communities result in a lower per cent of aggressive deer mice and in lower SNV seroprevalence (Dizney \& Denise Dearing, 
2016). A landscape-level adaptation of our model could help us understand how density and relative composition of 'bold' vs. 'shy' mice interact, along with the environment, to change hantavirus transmission dynamics.

Also, the resulting reproduction number distribution, with a mean of $R_{0}=1.3$ (Figure 5), is very close to computed values in other studies including Allen, McCormack, and Jonsson (2006) $\left(R_{0}=1.38\right)$, Gedeon, Bodelón, and Kuenzi $(2010)\left(R_{0}=1.28\right)$, and Luis, Douglass, Mills, and Bjørnstad (2010) where $R_{0}$ ranged from .19 to 5.73, with a mean of 1.27. Our reproduction numbers and resulting $R_{0}$ are an emergent property of the simulations, as they were not metrics we used when choosing the free parameter values.

The intervals of 3, 6 and 9 for aggression and viral shedding were chosen arbitrarily as a proxy and to prevent potential overfitting, when in reality, levels of aggression or virus infectivity are not on a discrete scale and have infinite possibilities. However, this simplified proxy of parameter values provided results very similar to Bagamian et al. (2012a), as well as other theoretical and empirical studies. With better data about variation in individual mouse aggression levels and viral shedding when infected, we can parameterize the model more accurately.

The model does not address effects of seasonality and breeding condition. Instead each mouse maintained their aggression level throughout the simulation. In reality, aggression levels may vary based on several factors, including time of year. Also, infection status may contribute to behavioural changes, which we did not account for. We also didn't take into account male and female differences. Finally, better data are needed to estimate parameters related to mouse behavior and individual mouse immune responses to SNV.

In the future, we would like to include seasonality and variation in male and female behavior in mice. Better understanding of deer mouse behavior and spatial movement patterns will lead to a model that can better approximate spread in the wild at larger scales and across seasons. A promising future direction of investigation is to apply this model to a large free-roaming population, then use the outputted results to inform faster and less- data-intensive ordinary differential equation and stochastic differential equation (ODE/SDE) models.

\section{Acknowledgements}

The content is solely the responsibility of the authors and does not necessarily represent the official views of the NSF. Special thanks to Mac Hyman for discussions about the project and model and for his ongoing support.

\section{Disclosure statement}

No potential conflict of interest was reported by the authors.

\section{Funding}

CM and MK were supported by NSF SEES Fellows [grant number CHE-1314029]. This work was assisted by attendance as a Short-term Visitor at the National Institute for Mathematical and Biological Synthesis, an Institute sponsored by the National Science Foundation through NSF [grant number DBI-1300426], with additional support from The University of Tennessee, Knoxville. This work was also supported in part by an NIH/NIGMS grant in the Models of Infectious Disease Agent Study (MIDAS) programme, [grant number U01-GM097661-01]. 


\section{References}

Abbott, K. D., Ksiazek, T. G., \& Mills, J. N. (1999). Long-term hantavirus persistence in rodent populations in central Arizona. Emerging Infectious Diseases, 5, 102-112.

Adler, F. R., Clay, C. A., \& Lehmer, E. M. (2008). The role of heterogeneity in the persistence and prevalence of Sin Nombre virus in deer mice. The American Naturalist, 172, 855-867.

Allen, L. J. S., McCormack, R. K., \& Jonsson, C. B. (2006). Mathematical models for hantavirus infection in rodents. Bulletin of Mathematical Biology, 68, 511-524.

Bagamian, K. H., Douglass, R. J., Alvarado, A., Kuenzi, A. J., Amman, B. R., Waller, L. A., \& Mills, J. N. (2012a). Population density and seasonality effects on Sin Nombre virus transmission in North American deermice Peromyscus maniculatus in outdoor enclosures. PLoS ONE, 7, 06 $1-11$.

Bagamian, K. H., Towner, J. S., Kuenzi, A. J., Douglass, R. J., Rollin, P. E., Waller, L. A., \& Mills, J. N. (2012b). Transmission ecology of Sin Nombre hantavirus in naturally infected North American deermouse populations in outdoor enclosures. PLOS ONE, 7, 10 1-10.

Bagamian, K. H., Towner, J. S., Mills, J. N., \& Kuenzi, A. J. (2013). Increased detection of Sin Nombre hantavirus RNA in antibody-positive deer mice from Montana, USA: Evidence of male bias in RNA viremia. Viruses, 5, 2320-2328.

Baumgardner, D. J., Ward, S. E., \& Dewsbury, D. A. (1980). Diurnal patterning of eight activities in 14 species of muroid rodents. Animal Learning \& Behavior, 8, 322-330.

Canadian Centre for Occupational Health and Safety (2008). Hantavirus: OSH answers. Retrieved January 25, 2016 from https://www.ccohs.ca/oshanswers/diseases/hantavir.html

Carver, S., Mills, J. N., Parmenter, C. A., Parmenter, R. R., Richardson, K. S., Harris, R. L., ... Luis, A. D. (2015). Toward a mechanistic understanding of environmentally forced zoonotic disease emergence: Sin Nombre hantavirus. BioScience, 65, 651-666.

Carver, S., Trueax, J. T., Douglass, R., \& Kuenzi, A. (2011). Delayed density-dependent prevalence of Sin Nombre virus infection in deer mice Peromyscus maniculatus in central and Western Montana. Journal of Wildlife Diseases, 47, 56-63.

CDC Hantavirus website. (2016, September 6). Retrieved from http://www.cdc.gov/hantavirus/

Clay, C. A., Lehmer, E. M., Previtali, A., \& Jeor, S. S. (2009). Contact heterogeneity in deer mice: Implications for Sin Nombre virus transmission. Proceedings of the Royal Society of London B: Biological Sciences, rspb-2008. doi:10.1098/rspb.2008.1693

Cline, B. J., Carver, S., \& Douglass, R. J. (2010). Relationship of human behavior within outbuildings to potential exposure to Sin Nombre virus in Western Montana, EcoHealth, 7, 389-393.

Dearing, M. D., Clay, C., Lehmer, E., \& Dizney, L. (2015). The roles of community diversity and contact rates on pathogen prevalence. Journal of Mammalogy, 96, 29-36.

Dizney, L., \& Dearing, M. D. (2013). The role of behavioural heterogeneity on infection patterns: Implications for pathogen transmission. Animal Behaviour, 86, 911-916.

Dizney, L., \& Dearing, M. D. (2016). Behavioural differences: A link between biodiversity and pathogen transmission. Animal Behaviour, 111, 341-347.

Douglass, R. J., Calisher, C. H., Wagoner, K. D., \& Mills, J. N. (2007). Sin Nombre virus infection of deer mice in Montana: Characteristics of newly infected mice, incidence, and temporal pattern of infection. Journal of Wildlife Diseases, 43, 12-22.

Douglass, R. J., Wilson, T., Semmens, W. J., Zanto, S. N., Bond, C. W., Van Horn, R. C., \& Mills, J. N. (2001). Longitudinal studies of Sin Nombre virus in deer mouse-dominated ecosystems of Montana. The American Journal of Tropical Medicine and Hygiene, 65, 33-41.

Eisenberg, J. F. (1962). Studies on the behavior of peromyscus maniculat us gambelii and peromyscus Californicus parasiticus. Behaviour, 19, 177-207.

Gavrilovskaya, I. N., Apekina, N. S., Bernshtein, A. D., Demina, V. T., Okulova, N. M., Myasnikov, Y. A, \& Chumakov, M. P. (1991). Pathogenesis of hemorrhagic fever with renal syndrome virus infection and mode of horizontal transmission of hantavirus in bank voles. In C. H. Calisher, Hemorrhagic fever with renal syndrome, tick-and mosquito-borne viruses (pp. 57-62). Vienna: Springer. 
Gedeon, T., Bodelón, C., \& Kuenzi, A. (2010). Hantavirus transmission in sylvan and peridomestic environments. Bulletin of Mathematical Biology, 72, 541-564.

Grimm, V., Berger, U., DeAngelis, D. L., Gary Polhill, J., Giske, J., \& Railsback, S. F. (2010). The ODD protocol: A review and first update. Ecological Modelling, 221, 2760-2768.

Jonsson, C. B., Figueiredo, L.T. M., \& Vapalahti, O. (2010). A global perspective on hantavirus ecology, epidemiology, and disease. Clinical Microbiology Reviews, 23, 412-441.

Kallio, E. R., Klingström, J., Gustafsson, E., Manni, T., Vaheri, A., Henttonen, H., ... Lundkvist, $\AA$, (2006). Prolonged survival of Puumala hantavirus outside the host: Evidence for indirect transmission via the environment. Journal of General Virology, 87, 2127-2134.

Kuenzi, A. J., Douglass, R. J., Bond, C. W., Calisher, C. H., \& Mills, J. N. (2005). Long-term dynamics of Sin Nombre viral RNA and antibody in deer mice in Montana. Journal of Wildlife Diseases, 41, $473-481$.

Linard, C., Tersago, K., Leirs, H., \& Lambin, E. F. (2007). Environmental conditions and Puumala virus transmission in Belgium. International Journal of Health Geographics, 6, 55. Retrieved from http://ij-healthgeographics.biomedcentral.com/articles/10.1186/1476-072X-6-55

Luis, A. D., Douglass, R. J., Mills, J. N., \& Bjørnstad, O. N. (2010). The effect of seasonality, density and climate on the population dynamics of Montana deer mice, important reservoir hosts for Sin Nombre hantavirus. Journal of Animal Ecology, 79, 462-470.

Luis, A. D., Douglass, R. J., Mills, J. N., \& Bjørnstad, O. N. (2014). Environmental fluctuations lead to predictability in Sin Nombre hantavirus outbreaks. Ecology, 96, 1691-1701.

Madhav, N. K., Wagoner, K. D., Douglass, R. J., \& Mills, J. N. (2007). Delayed density-dependent prevalence of Sin Nombre virus antibody in Montana deer mice Peromyscus maniculatus and implications for human disease risk. Vector-Borne and Zoonotic Diseases, 7, 353-364.

McCallum, H., Barlow, N., \& Hone, J. (2001). How should pathogen transmission be modelled? Trends in Ecology \& Evolution, 16, 295-300.

Millar, J. S. (1989). Reproduction and development. In G. L. J. Kirkland \& J. N. Layne (Eds.), Advances in the Study of Peromyscus (pp. 169-232). Lubbock: Texas Tech University Press.

Mills, J. N., Amman, B. R., \& Glass, G. E. (2010). Ecology of hantaviruses and their hosts in North America. Vector-Borne and Zoonotic Diseases, 10, 563-574.

Mills, J. N., \& Childs, J. E. (1998). Ecologic studies of rodent reservoirs: Their relevance for human health. Emerging Infectious Diseases, 4, 529-537.

Mills, J. N., Corneli, A., Young, J. C., Garrison, L. E., Khan, A. S., \& Ksiazek, T. G. (2002). Hantavirus pulmonary syndrome-United States: Updated recommendations for risk reduction. Morbidity and Mortality Weekly Report Recommendation and Reports RR, 51, 1-12.

Mills, J. N., Ksiazek, T. G., Peters, C. J., \& Childs, J. E. (1999). Long-term studies of hantavirus reservoir populations in the south western United States: A synthesis. Emerging Infectious Diseases, $5,135-142$.

Richardson, K. S., Kuenzi, A., Douglass, R. J., Hart, J., \& Carver, S. (2013). Human exposure to particulate matter potentially contaminated with Sin Nombre virus. EcoHealth, 10, 159-165.

Sauvage, F., Langlais, M., Yoccoz, N. G., \& Pontier, D. (2003). Modelling hantavirus in fluctuating populations of bank voles: The role of indirect transmission on virus persistence. Journal of Animal Ecology, 72, 1-13.

Turner, B. N., Iverson, S. L., \& Severson, K. L. (1983). Seasonal changes in open-field behavior in wild male meadow voles Microtus pennsylvanicus. Behavioral and Neural Biology, 39, 60-77.

Voutilainen, L., Sironen, T., Tonteri, E., Tuiskunen, A., Bäck, M., Razzauti, M., \& Lundkvist, Å. (2015). Life-long shedding of Puumala hantavirus in wild bank voles Myodes glareolus. Journal of General Virology, vir-0, 96, 1238-1247.

Wilensky, U. (1999). NetLogo. Evanston, IL: Center for Connected Learning and Computer-Based Modeling, North Western University.

Wilson, R. C., Vacek, T., Lanier, D. L., \& Dewsbury, D. A. (1976). Open-field behavior in muroid rodents. Behavioral Biology, 17, 495-506.

Woolhouse, M. E. J., Dye, C., Etard, J.-F., Smith, D. A., Charlwood, J. D., Garnett, G. P., ... Anderson, R. M. (1997). Heterogeneities in the transmission of infectious agents: Implications for the design of control programs. Proceedings of the National Academy of Sciences, 94, 338-342. 
Yanagihara, R., Gu, S. H., Arai, S., Kang, H. J., \& Song, J.-W. (2014). Hantaviruses: Rediscovery and new beginnings. Virus Research, 187, 6-14.

Yun, Z., \& others. (1999). Study on transmission of hemorrhagic fever with renal syndrome virus among experimental animals by aerosol. China Public Health, 2(10). 